\title{
Uncertainties in Secondary Fluorescence Correction in EPMA
}

\author{
Mathias Procop ${ }^{1}$ and Vasile-Dan Hodoroaba ${ }^{1}$
}

1. Federal Institute for Materials Research and Testing (BAM), Division 6.1 Surface Analysis and Interfacial Chemistry, 12200 Berlin, Germany

Secondary fluorescence is an inevitable effect that has to be taken into account in any algorithm for quantitative electron probe microanalysis (EPMA) as an additional correction [1,2]. Moreover, secondary fluorescence worsens spatial resolution of EPMA, as discussed once more in two recent papers [3,4]. Secondary fluorescence is excited both by characteristic radiation and by the X-ray continuum. In most cases the correction is small. There are, however, cases, e.g. the determination of low heavy metal concentration in a light matrix, where the contribution of secondary fluorescence exceeds $10 \%$ of the measured X-ray line intensity.

For secondary fluorescence correction the measured X-ray line intensity has to be divided by the correction factor $\left(1+\frac{I_{\text {flchar }}}{I_{p}}+\frac{I_{\text {flcont }}}{I_{p}}\right) \approx\left(1+\frac{I_{\text {flchar }}}{I_{p}}\right)\left(1+\frac{I_{\text {flcont }}}{I_{p}}\right)$ in order to get those intensity $I_{p}$, which is excited only by the primary electrons and enables the determination of specimen composition. $I_{\text {flchar }}$ and $I_{\text {flcont }}$ mean the calculated characteristic and continuums fluorescence intensities. In order to get the intensity of fluorescence radiation, the absorption of the exciting radiation in the specimen, the photoionization probability and the self-absorption of the emitted line must be calculated. This can be performed in a straightforward way [1,2]. The critical quantity is the X-ray yield of the exciting atoms in case of fluorescence by characteristic radiation and the bremsstrahlung yield of the specimen in case of continuum fluorescence. In the former case it is reasonable to apply the same physical model to calculate $I_{\text {flchar }}$ and $I_{p}$. In this way, scaling factor in the ionization cross section and stopping power will cancel, and the correction will not depend remarkably on the selected physical model. In case of continuums fluorescence, the yield is calculated by Kramers equation, and the intensity of the secondary continuum fluorescence can be written as

$$
I_{A}^{f l}=\frac{K}{2} Z_{\text {mean }} c_{A} \omega_{A} \frac{N}{A_{A}} \frac{\sin \psi}{\mu_{A}} \int_{E_{C}}^{E_{0}} \frac{E_{0}-E}{E} \sigma_{A}(E) \ln \left(1+\frac{\mu_{A}}{\mu(E) \sin \psi}\right) d E
$$

$Z_{\text {mean }}, c_{A}$ and $\omega_{A}$ stand for mean atomic number, mass concentration of atoms A with atomic weight $A_{A}$ and fluorescence yield. $N$ is the Avogadro number. $\mu(E)$ and $\mu_{A}$ denote the MACs for the exciting continuum radiation and for the fluorescence radiation emitted with the take-off angle $\psi \cdot \sigma_{A}(E)$ is the photoionization cross section for atoms $\mathrm{A}$, which is usually expressed by the MAC of the pure component A and the jump ratios. In the past, several simplifying assumptions were made to get an easy-to-handle expression for $\left(1+\frac{I_{\text {flcont }}}{I_{p}}\right)$, see e.g. [2], p.252 ff. Related uncertainties are overcome today by numerical integration. However, an uncertainty remains because different physical models can be applied to get $I_{p}$. This is shown for the continuum fluorescence correction in $\mathrm{Fe}$, Ni and Fe75Ni25 (Table 1). The example is given by Reed [2] using the Thomson Widdington law for the stopping power and is extended here applying different formulas for electron ionization cross section and stopping power to calculate $I_{p}$. XPP [5] and the original PROZA96 [6] give larger correction factors. This can be explained by the fact that calculated $I_{p}$ are somewhat underestimated as could be shown by comparison of calculated total emitted intensities of $\mathrm{K} \alpha$ lines with measurements performed with a calibrated spectrometer [7]. The best agreement between calculations and measurements could be achieved replacing the cross section formula applied by Bastin [6] with Casnati's formula [8]. The largest contribution to the uncertainty in continuum fluorescence 
corrections, however, comes from the constant $K$ in the Kramers equation. Only a few data for metal specimens can be found in literature ranging from $1.6 \times 10^{-6}$ to $3.2 \times 10^{-6} \mathrm{keV}^{-1}$ [9]. For the data in Table 1 own measurements with the calibrated spectrometer [7] were evaluated, giving $K=(3.0 \pm 0.2) \times 10^{-6} \mathrm{keV}^{-1}$. Fig. 1 shows as examples the measured and calculated background in $30 \mathrm{kV}$ spectra of $\mathrm{Zr}$ and GaP. More experimental data for $K$ are needed to reduce the uncertainty in continuum fluorescence correction.

\section{References:}

[1] K F J Heinrich, Electron Beam X-ray Microanalysis (van Nostrand, New York, 1981).

[2] S J B Reed, Electron Microprobe Analysis, 2nd ed. (Cambridge University Press, 1993)

[3] N W M Ritchie, Microsc Microanal 23 (2017), p. 1.

[4] B Buse, Microsc Microanal 24 (2018), p. 604.

[5] J-L Pouchou and F Pichoir in "Electron Beam Quantitation”, ed. K F J Heinrich and D E Newbury, (Plenum Press, New York), p. 31.

[6] G F Bastin, J M Dijkstra and H J M Heijligers, X-ray Spectrometry 27 (1998), p. 3.

[7] M Procop, Microsc Microanal 10 (2004), p. 481.

[8] E Casnati, A Tartari and C Baraldi, J Phys B 15 (1982), p. 155.

[9] M Green and V E Cosslett, Brit J Appl Phys (J Phys D) ser. 2, 2 (1968), p. 425.

[10] D Bote et al, Atomic Data Nuclear Data Tables 95 (2009), p. 871.

Table 1. Continuum fluorescence correction factors for the $\mathrm{K} \alpha$ line at $25 \mathrm{kV}$ and $40^{\circ}$ take-off angle in the Fe-Ni system. For own calculations $K=3.0 \times 10^{-6} \mathrm{keV}^{-1}$ was assumed, Reed [2] used $2.76 \times 10^{-6} \mathrm{keV}^{-1}$.

\begin{tabular}{|l|c|c|c|c|c|}
\hline $\begin{array}{l}\text { Element/ } \\
\text { Specimen }\end{array}$ & $\begin{array}{c}\text { Reed } \\
{[2], \text { p. 266 }}\end{array}$ & $\begin{array}{c}\text { XPP } \\
{[5]}\end{array}$ & $\begin{array}{c}\text { PROZA96 } \\
{[6]}\end{array}$ & $\begin{array}{c}\text { PROZA96 } \\
\text { Casnati [8] }\end{array}$ & $\begin{array}{c}\text { PROZA96 } \\
\text { Bote [10] }\end{array}$ \\
\hline Fe pure & 1.025 & 1.037 & 1.037 & 1.028 & 1.031 \\
\hline Fe in Fe75Ni25 & 1.018 & 1.038 & 1.038 & 1.029 & 1.032 \\
\hline Ni pure & 1.034 & 1.054 & 1.053 & 1.040 & 1.042 \\
\hline Ni in Fe75Ni25 & 1.019 & 1.036 & 1.036 & 1.027 & 1.030 \\
\hline
\end{tabular}
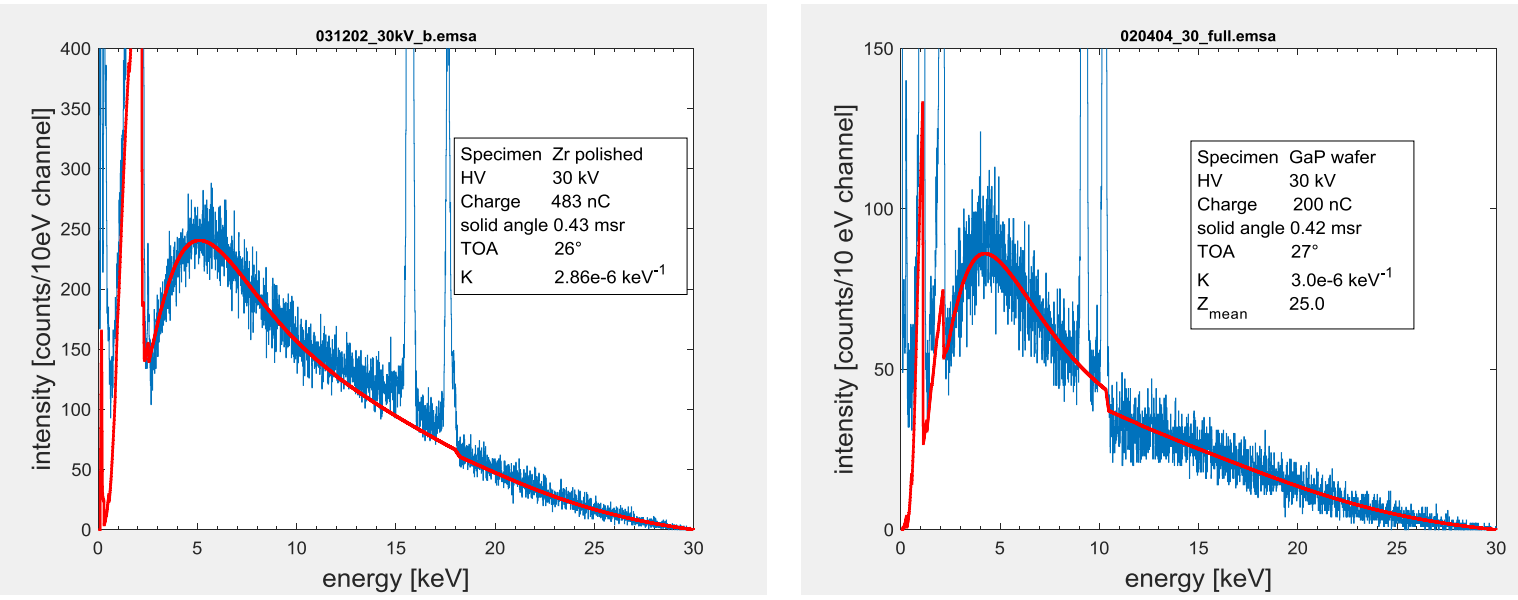

Figure 1. $30 \mathrm{kV}$ spectra of $\mathrm{Zr}$ (left) and GaP (right), measured with an efficiency calibrated spectrometer (counts/photon) and the calculated background (red) assuming $K=2.86 \times 10^{-6} \mathrm{keV}^{-1}$ for $\mathrm{Zr}$ and $K=3.0 \times 10^{-6}$ $\mathrm{keV}^{-1}$ for GaP. Kramers continuum was multiplied with Philibert's absorption correction and with the efficiency of the spectrometer to get the background (red). 